Adrian Heringer Pizzinga

\author{
Modelos em Espaço de Estado com \\ Restrições nas Componentes de Interesse: \\ Aplicações em Análise Dinâmica de Estilo \\ para Fundos de Investimento Brasileiros
}

Dissertação de Mestrado

Departamento de Engenharia Elétrica Programa de Pós-Graduação em Engenharia Elétrica 


\title{
Modelos em Espaço de Estado com Restrições nas Componentes de Interesse: Aplicações em Análise Dinâmica de Estilo para Fundos de Investimento Brasileiros
}

\author{
Dissertação de Mestrado \\ Dissertação apresentada como requisito parcial \\ para obtenção do título de Mestre pelo \\ Programa de Pós-Graduação em Engenharia \\ Elétrica da PUC-Rio.
}

Orientador: Cristiano Augusto Fernandes 
Ficha Catalográfica

Pizzinga, Adrian Heringer

Modelos em espaço de estado com restrições nas componentes de interesse: aplicações em análise dinâmica de estilo para fundos de investimento brasileiros / Adrian Heringer Pizzinga ; orientador: Cristiano Augusto C. Fernandes - Rio de Janeiro : PUC, Departamento de Engenharia Elétrica, 2004.

$131 \mathrm{f.} ; 30 \mathrm{~cm}$

Dissertação (mestrado) - Pontifícia Universidade Católica do Rio de Janeiro, Departamento de Engenharia Elétrica.

Inclui referências bibliográficas.

1. Engenharia Elétrica - Teses. 2. Modelos em espaço de estado. 3. Recursões de Kalman. 4. Metodologia de Doran. 5. Filtros não-lineares aproximados. 6. Análise dinâmica de estilo. I. Fernandes, Cristiano Augusto C. II. Pontifícia Universidade Católica do Rio de Janeiro. Departamento de Engenharia Elétrica. III. Título. 


\section{Modelos em Espaço de Estado com Restrições nas Componentes de Interesse: Aplicações em Análise Dinâmica de Estilo para Fundos de Investimento Brasileiros}

Dissertação apresentada como requisito parcial para obtenção do título de Mestre pelo Programa de Pós-Graduação em Engenharia Elétrica da PUC-Rio.

Cristiano Augusto Coelho Fernandes Orientador

Departamento de Engenharia Elétrica - PUC-Rio

Álvaro Lima Veiga Filho Departamento de Engenharia Elétrica - PUC-Rio

Carlos S. Kubrusly Departamento de Engenharia Elétrica - PUC-Rio

Marcelo Cunha Medeiros Departamento de Economia - PUC-Rio

Marcos Azevedo da Silveira Suplente Departamento de Engenharia Elétrica - PUC-Rio

Nei Carlos dos Santos Rocha Instituto de Matemática e Estatística - UFRJ

Prof. José Eugenio Leal Coordenador Setorial do Centro

Técnico Científico - PUC-Rio

Rio de Janeiro, 18 de março de 2004 


\section{Agradecimentos}

Antes de tudo, gostaria de agradecer especialmente à minha família pela tolerância dedicada a mim e às minhas atividades acadêmicas no transcorrer desses últimos anos.

Agradeço também ao meu orientador, professor e amigo Cristiano Fernandes pelo apoio, confiança e reconhecimento de meu esforço como Mestrando deste departamento.

Dedico também um agradecimento à banca examinadora desta Dissertação. Sem entrar em detalhes, há membros nela compreendidos que foram, são e sempre serão meus eternos professores e amigos. Eu devo considerável parte de minha qualificação a estas pessoas tão especiais.

Quero agradecer também a André Gustavo Guimarães, a Sergio Contreras e a Washington Junger pela valiosíssima ajuda nas implementações dos modelos desta Dissertação.

Gostaria de agradecer aos colegas Raphael Cruz e Gustavo Raposo pelos valiosos comentários e pelas ricas discussões a respeito dos fundamentos teóricos referentes aos modelos propostos pela Dissertação.

Agradeço ao amigo Ezequiel Zibecchi, vulgo "Anbídim”, pelos conhecimentos transmitidos sobre o mercado brasileiro de fundos de investimentos durante o tempo em que fomos colegas de trabalho na Atlantic Rating. Este knowhow, por mim adquirido, foi imprescindível para as análises interpretativas das aplicações contempladas na Dissertação.

Um salve para o amigo Henri, o grande "rotacionador de eixos cartesianos", pela torcida sincera e disposição para me ajudar. Henri, você não entendeu? Vou lhe explicar. x, y e z . Pegou, rodou...

Agradeço ao amigo Luiz Felipe ("Felipão") pela ajuda na confecção da Dissertação, pelas orientações de como eu deveria proceder com minhas obrigações formais com o Departamento e, acima de tudo... pelas maravilhosas gestão e manutenção do Laboratório de Estatística Computacional (LEC), o qual vem sido por ele gerenciado desde a renúncia de seu ex-presidente André Gustavo Guimarães. 
Agradeço ao amigo "cidadão de bem” Savano Souza Pereira (nãããão...) pela ajuda na confecção dos artigos gerados da Dissertação.

Gostaria de agradecer também ao parceiro Rodrigo Lage, "cabeção" (assim como o Felipão) e atual estagiário de um importante político da Bahia, que sempre torceu pelo meu sucesso e que sabe da reciprocidade desta torcida. Agora não posso falar mais, pois estou... "naquele looonce...".

Não posso me esquecer das torcidas dos meus amigos Rafael Martins, futuro governador do Estado do Rio de Janeiro, e Eduardo Campos, futuro presidente de honra da ENCE. Rafael e Eduardo, não se animem tanto, pois este futuro pode estar ainda muito distante!

Agradeço também pelos incentivos de Blanca, Tereza, Isolina, Bruno e Vivian. Aliás, Tereza, para você que sempre perguntava: finalmente "convergiu"!

Dedico também um agradecimento especial (sem citar nomes, pois posso esquecer alguém...) aos meus amigos que estiveram presentes e me apoiaram, de alguma forma, durante meu Mestrado. Vocês sabem quem são.

Finalmente, quero agradecer à PUC por ter me acolhido (e ainda me acolher) tão bem durante este período de Mestrado e às instituições incentivadoras de pesquisa CAPES e FAPERJ pelo importante auxílio financeiro. 


\section{Resumo}

PIZZINGA, Adrian Heringer; FERNANDES, Cristiano Augusto Coelho (Orientador). Modelos em Espaço de Estado com Restrições nas Componentes de Interesse: Aplicações em Análise Dinâmica de Estilo para Fundos de Investimento Brasileiros. Rio de Janeiro, Março, 2004. 131p. Dissertação de Mestrado - Departamento de Engenharia Elétrica, Pontifícia Universidade Católica do Rio de Janeiro.

Esta Dissertação procura, sob um enfoque freqüentista, discutir tecnologias para que se imponham restrições no processo de estimação de componentes não observáveis associadas a um modelo em Espaço de Estado (EE) arbitrário. O escopo do texto abrange desde procedimentos propostos pioneiramente por Howard Doran para restrições de igualdade, lineares e/ou não lineares, invariantes ou variantes no tempo, em modelos em EE lineares, até a adoção e o ajuste de estruturas mais delicadas, como os modelos em EE não lineares. Entende-se que estes últimos se constituem em uma alternativa relevante, caso seja requerida, por exemplo, a imposição de restrições de desigualdade. Técnicas e estratégias de implementação são apresentadas, debatidas e comparadas, incluindo-se também o processo de estimação de parâmetros desconhecidos e a questão de diagnósticos. Ao final, são apresentados exercícios empíricos com base nas tecnologias discutidas. Os modelos propostos para esta ilustração visam à realização da análise dinâmica de estilo baseado no retorno para carteiras de investimento brasileiras (a versão estática desses modelos fora introduzida por William Sharpe, para carteiras norte-americanas), os quais devem, eventualmente, abranger dois tipos de restrições nas componentes de interesse, quais sejam, um de igualdade e outro de desigualdade.

\section{Palavras-chave}

Modelos em Espaço de Estado, Componentes de Interesse, Recursões de Kalman, Inicialização Exata, Metodologia de Doran, Filtros Não Lineares Aproximados, Análise Dinâmica de Estilo. 


\section{Abstract}

PIZZINGA, Adrian Heringer; FERNANDES, Cristiano Augusto Coelho (Advisor). State Space Models with Restrictions in Components of Interest: Applications in Dynamic Style Analysis for Brazilian Investment Funds. Rio de Janeiro, March, 2004. 131p. MSc. Dissertation - Departamento de Engenharia Elétrica, Pontifícia Universidade Católica do Rio de Janeiro.

This Dissertation aims, in a frequentist way, to discuss technologies for imposing restrictions in non-observable components associated with an arbitrary State Space (SS) model. The text scope ranges from procedures proposed originally by Howard Doran for equality, linear or non-linear, time invariant or time varying restrictions in a linear SS model, to adoption and estimation of more complicated structures like non-linear SS models. It is understood that these last ones are a relevant alternative, in cases of, for instance, inequality restrictions requirement. Implementation techniques and strategies are given, debated and compared, also including unknown parameters estimation and diagnostics analysis. At the end, empirical exercises are presented based on discussed methodologies. The proposed models for this illustration aim at dynamic return based style analysis for Brazilian investment portfolios (the static version of these models had been introduced by William Sharpe, for American portfolios), which shall eventually satisfy two kinds of restrictions on components of interest, namely one of equality and other of inequality.

\section{Keywords}

State Space Models, Components of Interest, Kalman Recursions, Exact Initialization, Doran's Methodology, Approximated Non-Linear Filters, Dynamic Style Analysis. 


\section{Sumário}

1. Introdução 10

1.1 - Considerações Iniciais 10

1.2. - Problema Central 11

1.3 - Objetivos da Dissertação 12

1.4. - Estrutura da Dissertação 13

2. Modelos em Espaço de Estado Lineares: Formulação Geral 16

2.1 - Definição Geral de um Modelo Linear $\quad 16$

2.2 - Definição de Componente 19

3. Filtro, Previsor e Suavizador de Kalman 21

3.1- As Três Formas Básicas de Estimação do Vetor de Estado 21

3.2 - Previsão $\quad 22$

3.3 - Atualização 23

$\begin{array}{ll}3.4 \text { - Suavização } & 25\end{array}$

3.5 - Hierarquia de Previsão para as Três Formas de Estimação 27

3.6 - Inicialização do Filtro de Kalman 28

3.6.1 - Inicialização Difusa Aproximada 30

3.6.2 - Inicialização Difusa Exata 31

4. Imposição de Restrições Lineares: A Metodologia de Doran 33

4.1 - Restrições nos Filtrados 33

4.2 - Restrições nos Suavizados $\quad 35$

4.3 - Restrição nos Previstos 38

4.4 - Eficiência da Metodologia de Doran em Relação à Estimação Irrestrita 38

5. Imposição de Restrições Não Lineares: A Metodologia de Doran

Estimada $\quad 42$

5.1 - O Algoritmo Original 42

5.2 - Proposta de Alteração no Algoritmo 43

5.3 - Restrições de Desigualdade 44

6. Modelos em Espaço de Estado Não Lineares e Filtros Não Lineares Aproximados $\quad 45$

6.1 - Definição Geral de um Modelo Não Linear $\quad 45$

6.2 - O Filtro de Kalman Estendido $\quad 49$

6.2.1 - As Recursões do Filtro de Kalman Estendido 50

6.2.2 - Propriedades do Filtro de Kalman Estendido 51

6.2.2.1 - Vantagens $\quad 52$

6.2.2.2 - Desvantagens 53

6.3 - O Filtro de Segunda Ordem $\quad 55$

6.3.1 - Recursões do Filtro de Segunda Ordem $\quad 55$

6.3.2 - Propriedades do Filtro de Segunda Ordem 56

6.4 - O Filtro de Simulação de Monte Carlo 56

6.4.1 - Recursões do Filtro de Simulação de Monte Carlo 57

6.4.2 - Propriedades do Filtro de Simulação de Monte Carlo 62

7. Estimação de Parâmetros Desconhecidos e a Questão dos Diagnósticos 65

7.1 - Função de Log Verossimilhança para Modelos Lineares 65

$\begin{array}{ll}7.1 .1 \text { - Inicialização Não Difusa } & 65\end{array}$ 
7.1.2 - Inicialização Difusa 66

7.1.2.1 - Inicialização Difusa Aproximada 66

7.1.2.2 - Inicialização Difusa Exata 67

7.2 - Função de Quasi Log Verossimilhança para Modelos Lineares 68

7.3 - Propriedades Assintóticas dos Estimadores de (Quasi) Máxima

Verossimilhança

7.4 - Função de Log Verossimilhança Aproximada para Modelos Não
Lineares Via Tratamento Aproximado

$\begin{array}{ll}7.5 \text { - Diagnósticos } & 71\end{array}$

$\begin{array}{ll}\text { 7.5.1- Análise de Resíduos } & 71\end{array}$

$\begin{array}{ll}\text { 7.5.2 - Análise do Poder Preditivo } & 73\end{array}$

8. Aplicações: Modelos Dinâmicos para Análise de Estilo Baseada no Retorno $\quad 74$

8.1 - Análise de Estilo Baseada no Retorno $\quad 75$

$\begin{array}{ll}\text { 8.1.1 - Motivação da Técnica } & 75\end{array}$

8.1.2 - Modelo de Fatores para Classes de Ativos 77

$\begin{array}{ll}8.1 .3 \text { - Implementação } & 80\end{array}$

8.1.4 - Defeitos Naturais da Técnica $\quad 80$

8.2 - Uma Proposta para Análise Dinâmica de Estilo 81

8.2.1 - Formulação em EE: Equação do Estado 82

8.2.2 - Formulação em EE: Equação das Medidas 83

8.3 - Análises Dinâmicas de Estilo para Fundos de Investimento Brasileiros $\quad 86$

8.3.1 - Primeiro Exercício: Fundo de Ações 88

8.3.1.1 - Análise Dinâmica de Estilo Fraca 88

8.3.1.2 - Análise Dinâmica de Estilo Semi-Forte 92

8.3.1.3 - Análise Dinâmica de Estilo Forte 94

8.3.2 - Segundo Exercício: Fundo Cambial 101

8.3.2.1 - Análise Dinâmica de Estilo Fraca 102

$\begin{array}{ll}\text { 8.3.2.2 - Análise Dinâmica de Estilo Semi-Forte } & 104\end{array}$

8.3.2.3 - Análise Dinâmica de Estilo Forte 107

$\begin{array}{ll}\text { 8.3.3 - Terceiro Exercício: Carteira Simulada } & 114\end{array}$

9. Considerações Finais sobre a Pesquisa 117

9.1 - Conclusões sobre as Metodologias Discutidas 117

9.2 - Conclusões sobre a Análise Dinâmica de Estilo 119

$\begin{array}{lr}9.3 \text { - Conclusões sobre Estudos Futuros } & 120\end{array}$

$\begin{array}{lr}\text { Bibliografia } & 123\end{array}$

$\begin{array}{lr}\text { Bibliografia complementar } & 128\end{array}$

Anexo 1 - Estimativas dos parâmetros desconhecidos para o fundo

$\begin{array}{ll}\text { Bradesco II FIA } & 130\end{array}$

Anexo 2 - Estimativas dos parâmetros desconhecidos para o fundo HSBC FIF Cambial 